\title{
The importance of individualized article-specific metrics for evaluating research productivity Kuan-Teh Jeang
}

Address: National Institutes of Health, Bethesda, MD, USA

Email: Kuan-Teh Jeang -kj7e@nih.gov

Published: 16 September 2009

Retrovirology 2009, 6:82 doi:10.1/86/1742-4690-6-82

This article is available from: http://www.retrovirology.com/content/6/I/82

2009 Jeang; licensee BioMed Central Ltd.

This is an Open Access article distributed under the terms of the Creative Commons Attribution License (http://creativecommons.org/licenses/by/2.0), which permits unrestricted use, distribution, and reproduction in any medium, provided the original work is properly cited.

Received: 15 September 2009

Accepted: 16 September 2009

\begin{abstract}
This editorial discusses the rationale for using article-specific rather than journal-specific metrics for evaluating highly published authors.
\end{abstract}

\section{Editorial}

Mark Patterson of PLoS (Public Library of Science) recently wrote an online piece http://www.plos.org/cms/ node/478 on how to measure impact where it matters. Patterson makes an important point that one should focus on article specific metrics when evaluating a published paper rather than relying "on the name and the impact factor (IF) of the journal in which the work is published". In the past, it was not always easy to assess quickly and accurately the citations to individually published articles. Today, many electronic tools (e.g. ISI Web of Science, Scopus, Google Scholar) exist that can accomplish this task facilely and reliably. Because there are inherent shortcomings to how a journal's IF is calculated and because of the rather poor representativeness of the IF for the citations to individual articles $[1,2]$, institutions and peerreview bodies should be encouraged strongly to employ article-specific measures in preference to journal IFs in evaluations.

Article-specific citations are often not used properly in evaluating published authors. For example, in some circles, it has become fashionable to create lists of "highly cited" scientists in various fields (e.g. http://isihighly cited.com/; highly cited in immunology, highly cited in microbiology, highly cited in molecular biology and genetics etc...). In some respects, these lists could be useful conveniences, provided that the users understand clearly how they are generated and what they mean (and do not mean). One could assume that "highly cited in microbiology" is based on article specific-citations. In fact, this would be a mistaken assumption because the listing is actually based on journal-specific data. What does this mean? By way of explanation, let's consider a hypothetical illustration. If John Smith were an author of 10 papers on HIV-1 published in Cell or the Journal of Biological Chemistry (which are not counted by ISIHighlyCited as microbiology journals) and if these 10 papers were cited cumulatively 1,000 times over a specified duration, then Smith's citation counts based on these papers for purposes of "highly cited in microbiology" would be 0 . On the other hand, if the exactly same 10 Smith papers on HIV were unsuccessful in initial submissions to Cell or the Journal of Biological Chemistry, but were subsequently successfully published in the Journal of Virology, Retrovirology, or Virology (all counted as microbiology journals), then the 1,000 citations to these papers would add 1,000 counts to Smith's ranking for purposes of "highly cited in microbiology". So, here is an example where journal-specific metrics trump article-specific measures. In order to be "highly cited in microbiology", what one publishes (i.e. article-specific content on HIV) counts not unless it is published in a journal deemed as "microbiology" (i.e. a journal-specific metric). Thus, this illustration shows that 
Table I: $\mathrm{H}$-index and citation frequencies of selected Retrovirology editorial board members.

\begin{tabular}{|c|c|c|c|c|c|c|c|}
\hline Title & Name & $\begin{array}{l}\text { Role within Retro- } \\
\text { virology }\end{array}$ & Institution & City & Country & $H$ index & $\begin{array}{l}\text { Total times cited } \\
\text { since } 1996\end{array}$ \\
\hline Dr. & Kuan-Teh Jeang & Editor-in-Chief & $\mathrm{NIH}$ & Bethesda & USA & 46 & 9799 \\
\hline Dr. & Monsef Benkirane & Editor & CNRS & Montpellier & France & 23 & 2210 \\
\hline Dr. & Ben Berkhout & Editor & Academic Med. Ctr & Amsterdam & the Netherlands & 40 & 6925 \\
\hline Dr. & Andrew Lever & Editor & $\begin{array}{l}\text { Cambridge } \\
\text { University }\end{array}$ & Cambridge & UK & 19 & 2065 \\
\hline Dr. & Mark Wainberg & Editor & McGill University & Montreal & Canada & 40 & 10058 \\
\hline Dr. & Masahiro Fujii & Editor & Niigata University & Niigata & Japan & 21 & 2186 \\
\hline Dr. & Michael Lairmore & Editor & $\begin{array}{l}\text { Ohio State } \\
\text { University }\end{array}$ & Columbus & USA & 21 & 2226 \\
\hline Dr. & Michael Bukrinsky & Ed Board & $\begin{array}{l}\text { George Washington } \\
\text { Univ }\end{array}$ & Washington DC & USA & 26 & 5218 \\
\hline Dr. & Dong-yan Jin & Ed Board & Hong Kong U & Hong Kong & China & 25 & 2675 \\
\hline Dr. & Klaus Strebel & Ed Board & $\mathrm{NIH}$ & Bethesda & USA & 27 & 4395 \\
\hline Dr. & Tom J. Hope & Ed Board & U. Illinois & Chicago & USA & 27 & 4730 \\
\hline Dr. & Stephane Emiliani & Ed Board & Cochin Institute & Paris & France & 19 & 2061 \\
\hline Dr. & Patrick Green & Ed Board & $\begin{array}{l}\text { Ohio State } \\
\text { University }\end{array}$ & Columbus & USA & 19 & 1050 \\
\hline Dr. & Mauro Giacca & Ed Board & Int. Ctr. Genetics & Trieste & Italy & 38 & 5795 \\
\hline Dr. & Olivier Schwartz & Ed Board & Institut Pasteur & Paris & France & 31 & 5209 \\
\hline Dr. & Leonid Margolis & Ed Board & $\begin{array}{l}\text { National Inst Child } \\
\text { Health }\end{array}$ & Bethesda & USA & 23 & 2028 \\
\hline Dr. & Fatah Kashanchi & Ed Board & $\begin{array}{l}\text { George Washington } \\
\text { U. }\end{array}$ & Washington DC & USA & 27 & 2725 \\
\hline Dr. & Masao Matsuoka & Ed Board & Kyoto University & Kyoto & Japan & 29 & 3834 \\
\hline Dr. & Naoki Mori & Ed Board & $\begin{array}{l}\text { University of the } \\
\text { Ryukyus }\end{array}$ & Okinawa & Japan & 28 & 3375 \\
\hline Dr. & Chou-Zen Giam & Ed Board & $\begin{array}{l}\text { Uniform Services } \\
\text { Med School }\end{array}$ & Bethesda & USA & 16 & 1698 \\
\hline Dr. & David Derse & Ed Board & $\mathrm{NCl}$ & Frederick & USA & 15 & 1828 \\
\hline Dr. & Tatsuo Shioda & Ed Board & Osaka Univ & Osaka & Japan & 24 & 2110 \\
\hline Dr. & John Semmes & Ed Board & $\begin{array}{l}\text { Eastern Virginia Med } \\
\text { College }\end{array}$ & Norfolk & USA & 29 & 3416 \\
\hline Dr. & Anne Gatignol & Ed Board & McGill Univ. & Montreal & Canada & 17 & 1542 \\
\hline
\end{tabular}


Table I: $\mathbf{H}$-index and citation frequencies of selected Retrovirology editorial board members. (Continued)

\begin{tabular}{|c|c|c|c|c|c|c|c|}
\hline Dr. & Rogier Sanders & Ed Board & Academic Med. Ctr & Amsterdam & the Netherlands & 13 & 955 \\
\hline Dr. & Chen Liang & Ed Board & McGill Univ. & Montreal & Canada & 19 & 976 \\
\hline Dr. & Finn Skou Pedersen & Ed Board & University of Aarhus & Aarhus & Denmark & 19 & 1498 \\
\hline Dr. & Renaud Mahieux & Ed Board & Pasteur Int. & Paris & France & 24 & 1489 \\
\hline Dr. & Neil Almond & Ed Board & NIBSC & Potters Bar & UK & 15 & 1370 \\
\hline Dr. & Stephen P. Goff & Ed Board & Columbia University & New York & USA & 44 & $|485|$ \\
\hline Dr. & Johnson Mak & Ed Board & $\begin{array}{l}\text { Burnet Inst. Med. } \\
\text { Research }\end{array}$ & Victoria & Australia & 17 & 1679 \\
\hline Dr. & Christine Kozak & Ed Board & $\mathrm{NIH}$ & Bethesda & USA & 29 & 7814 \\
\hline Dr. & Greg Towers & Ed Board & Univ. College & London & UK & 17 & 1558 \\
\hline Dr. & Eric Cohen & Ed Board & Univ. Montreal & Montreal & Canada & 37 & 7047 \\
\hline Dr. & Warner Greene & Ed Board & UCSF & San Francisco & USA & 42 & 11011 \\
\hline Dr. & Jean-luc Darlix & Ed Board & U. Lyon & Lyon & France & 33 & 6070 \\
\hline Dr. & Eric Freed & Ed Board & $\mathrm{NCl}$ & Frederick & USA & 31 & 4906 \\
\hline Dr. & Toshiki Watanabe & Ed Board & Univ. of Tokyo & Tokyo & Japan & 24 & 2576 \\
\hline Dr. & Mari Kannagi & Ed Board & $\begin{array}{l}\text { Tokyo Med and } \\
\text { Dental U }\end{array}$ & Tokyo & Japan & 17 & 1474 \\
\hline Dr. & Frank Kirchhoff & Ed Board & University of Ulm & Ulm & Germany & 34 & 5478 \\
\hline Dr. & Jennifer Nyborg & Ed Board & Colorado State U & Fort Collins & USA & 18 & $|67|$ \\
\hline Dr. & $\begin{array}{l}\text { Akifumi Takaori- } \\
\text { Kondo }\end{array}$ & Ed Board & Kyoto University & Kyoto & Japan & 14 & 718 \\
\hline Dr. & Marc Sitbon & Ed Board & CNRS & Montpellier & France & 13 & 814 \\
\hline Dr. & Paul Gorry & Ed Board & $\begin{array}{l}\text { MacFarlane Burnet } \\
\text { Institute }\end{array}$ & Melbourne & Australia & 16 & 835 \\
\hline Dr. & David Harrich & Ed Board & $\begin{array}{l}\text { Queensland Inst } \\
\text { Medical Res. }\end{array}$ & Brisbane & Australia & 12 & 1063 \\
\hline Dr. & Susan Marriott & Ed Board & Baylor & Houston & USA & 15 & 1102 \\
\hline Dr. & Alan Cochrane & Ed Board & U Toronto & Toronto & Canada & 11 & 1191 \\
\hline Dr. & Yiming Shao & Ed Board & China CDC & Beijing & China & 14 & 1123 \\
\hline Dr. & Vinayaka Prasad & Ed Board & $\begin{array}{l}\text { Albert Einstein } \\
\text { College Medicine }\end{array}$ & New York & USA & 19 & 1239 \\
\hline Dr. & Roger Pomerantz & Ed Board & Tibotec & Yardley & USA & 34 & 6912 \\
\hline
\end{tabular}


Table I: H-index and citation frequencies of selected Retrovirology editorial board members. (Continued)

\begin{tabular}{|c|c|c|c|c|c|c|c|}
\hline Dr. & Li Wu & Ed Board & $\begin{array}{l}\text { Medical College } \\
\text { Wisconsin }\end{array}$ & Milwaukee & USA & 30 & 5617 \\
\hline Dr. & $\begin{array}{l}\text { Anne-Mieke } \\
\text { Vandamme }\end{array}$ & Ed Board & $\begin{array}{l}\text { Rega Inst. and Univ } \\
\text { Hospitals }\end{array}$ & Leuven & Belgium & 35 & 4994 \\
\hline Dr. & Alan Engelman & Ed Board & Harvard Univ. & Boston & USA & 25 & 4070 \\
\hline Dr. & Paul Clapham & Ed Board & Univ. Massachusetts & Worcester & USA & 30 & 6495 \\
\hline Dr. & Vinay Pathak & Ed Board & $\mathrm{NCl}$ & Frederick & USA & 25 & 1951 \\
\hline Dr. & Jeremy Luban & Ed Board & Univ. Geneva & Geneva & Switzerland & 29 & 4469 \\
\hline
\end{tabular}

ratings based on journal-specific data that do not properly integrate article-specific measures can be misleading when used to rate scientists. For retrovirologists, Retrovirology has emphasized consistently the use of person-specific measures of H-index [3] and total citations. Indeed, annually for the past three years, these data have been presented, using the Scopus data base http:// www.scopus.com, for selected Retrovirology editorial board members (see Table 1) [1,2].

Finally, one should not overlook the merits of awards and prizes in evaluating highly accomplished colleagues. Awards/prizes can come in two flavors; one as "leading" and the other as "lagging" indicators of scientific potential/productivity. For example, "life-time achievement" awards would be a "lagging" measure of one's achievements, while a "young" investigator prize might be a "leading" indicator of future potential. Retrovirology annually awards a "Retrovirology Prize" to a mid-career scientist $[4,5]$. The Prize aims to recognize "lagging" and "leading" benchmarks. It rewards the past achievements of a scientist who is in his/her mid-career and who still has substantial lead-time to accomplish future breakthrough research in retrovirology [5-8]. With this editorial, this year's nomination period for the 2009 Retrovirology Prize to recognize a retrovirologist for non-HIV-retrovirology research is open. The nomination period will close on October 31, 2009. The rules for nomination and the selection procedures remain the same as in past years $[9,10]$. Interested individuals can direct email inquiries to editorial@retrovirology.com.

\section{Authors' contributions}

KTJ wrote this editorial.

\section{Acknowledgements}

I thank Andrew Dayton and Mark Wainberg for critically reading this editorial and Daniel Schmidt for assistance in preparing Table I.

\section{References}

I. Jeang KT: Impact factor, $\mathbf{H}$ index, peer comparisons, and Retrovirology: is it time to individualize citation metrics? Retrovirology 2007, 4:42.

2. Jeang KT: H-index, mentoring-index, highly-cited and highlyaccessed: how to evaluate scientists? Retrovirology 2008, 5: 106.

3. Hirsch JE: Does the $\mathbf{H}$ index have predictive power? Proc Natl Acad Sci USA 2007, 104:19193-19198.

4. Jeang KT: Life after 45 and before 60: the Retrovirology Prize. Retrovirology 2005, 2:26.

5. Jeang KT: The 2008 Retrovirology Prize: Ben Berkhout and his RNA world. Retrovirology 2008, 5: I I 3.

6. Jeang KT: Small philanthropy and big science: the RETROVIROLOGY prize and Stephen P. Goff. Retrovirology 2005, 2:43.

7. Lever AM: Science--a life fully lived: Joe Sodroski wins the 2006 Retrovirology Prize. Retrovirology 2006, 3:45.

8. Boris-Lawrie K: Bridging fundamental RNA biology, retroviral replication, and oncogenesis: Karen Beemon wins the 2007 Retrovirology Prize. Retrovirology 2007, 4:88.

9. Jeang KT: The young, not-so-young, and the 2007 Retrovirology Prize: call for nominations. Retrovirology 2007, 4:64.

10. Jeang KT: Recognizing mid-career productivity: the 2008 Retrovirology Prize, call for nomination. Retrovirology 2008, 5:80.

Publish with Bio Med Central and every scientist can read your work free of charge

"BioMed Central will be the most significant development for disseminating the results of biomedical research in our lifetime. "

Sir Paul Nurse, Cancer Research UK

Your research papers will be:

- available free of charge to the entire biomedical community

- peer reviewed and published immediately upon acceptance

- cited in PubMed and archived on PubMed Central

- yours - you keep the copyright

Submit your manuscript here:

http://www.biomedcentral.com/info/publishing_adv.asp
BioMedcentral 